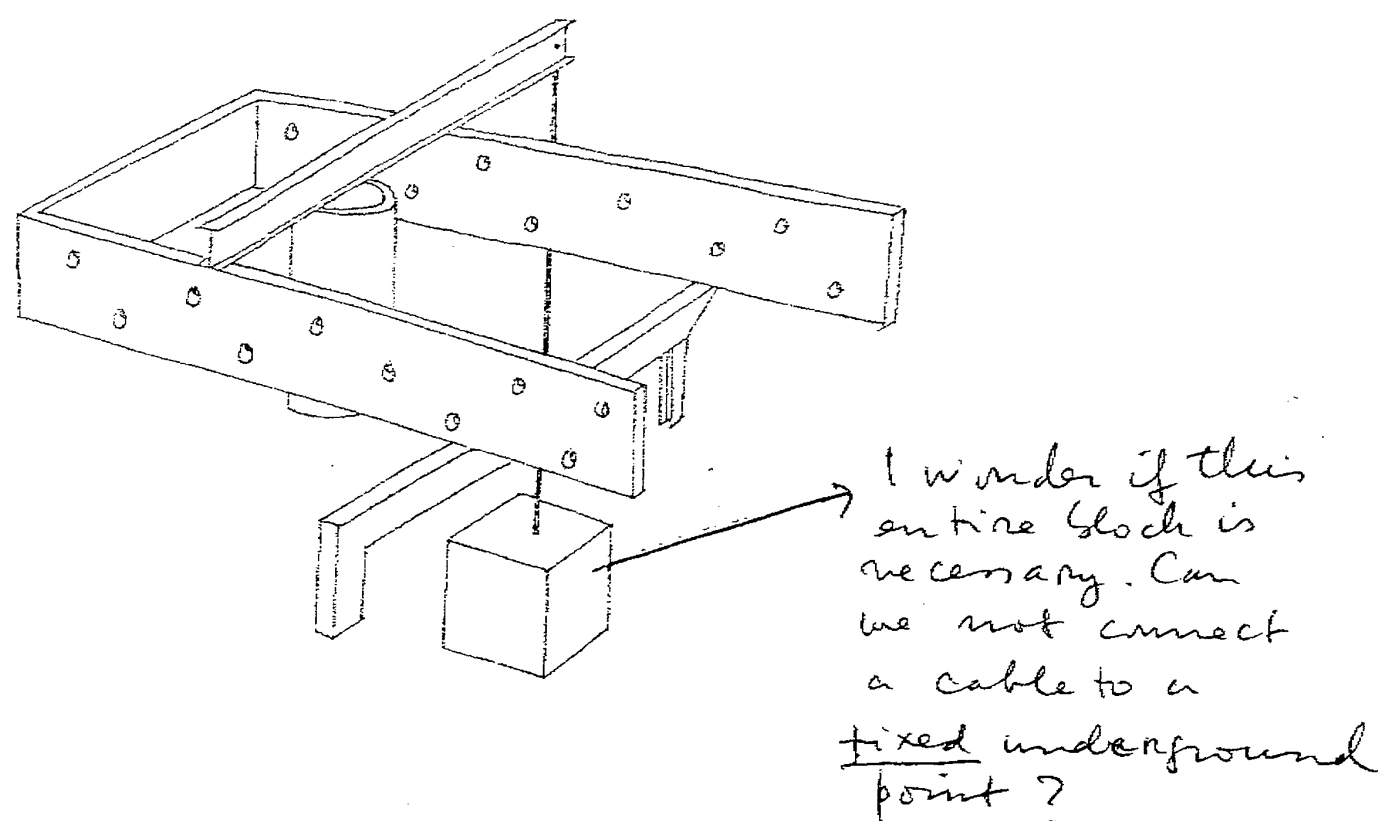

$\wedge$ FIG. 1. Croquis de esquema estructural con anotación de R.Koolhaas.
Fax de obra 16/03/1996

\section{Transcripción, intuición, mediación}

\section{Álvaro Moreno Hernández}

Recibido 2016.03.16 ::.: Aceptado 2016.04.16 DOI: 10.5821/palimpsesto.15.4813

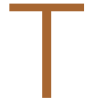

ranscribir remite a copiar, a hacerlo de un medio a otro y a hacerlo literalmente, lo cual implica conocer el código que permite esa equivalencia. Habla de un proceso, si no dirigido, al menos, reglado. De una transferencia, de una elaboración o de un pensamiento lógico.

La intuición se sitúa en otro nivel del conocimiento. No en el de la lógica sino en el de la revelación, el de la presentación evidente a la conciencia. Un acto más allá de la lógica en un ámbito interno, difícilmente comunicable o expresable con palabras, asociado al pensamiento analógico.

En la creación se manifiesta con intensidad esta dualidad del pensamiento y, en la arquitectura, el proyecto se convierte en el primer campo de batalla en el que se materializa la disputa entre ambas posiciones.

Se transcriben las intenciones de lo que se proyecta en elementos arquitectónicos, sometidos a la lógica de la disciplina (o subvirtiéndola). Como documento, el proyecto anuncia la posibilidad, un hacer verosímil. Será la obra, contaminada de la realidad material y objetiva de la ejecución, la que ensaye una nueva transcripción para presentar las intuiciones primeras, concluyendo la acción del proyectar o del querer llegar a ser.

De esta dualidad entre razón y emoción, técnica y poética (o transcripción e intuición), habla brillantemente Francisco Javier Sáenz de Oíza apoyándose en Le Corbusier y su definición de arquitectura.

El acierto de esta interpretación reside en la identificación de estos términos como complementarios y no como excluyentes. Ambos son necesarios, pero deben equilibrarse en la forma que necesite cada proyecto.

La misión del arquitecto, por tanto, será mediar entre ambas posturas (no por equidistancia, sino por equivalencia) para abrir el espacio de la arquitectura.

"[...] lo primero que deberíamos descubrir es, en qué medida, la durabilidad de la arquitectura, como hecho sustancial, humano, profundo y entrañable, conmovedor, y la (dijéramos) cotidianeidad de las técnicas, son compatibles en un mismo edificio para hacer que el uno no decaiga en función del otro."

(SÁENZ DE OÍZA F. J., Presentación del curso académico 91-92, 1991)

Así, la asignación que se podría hacer entre transcripción y mera construcción en el Le Corbusier de "Hacia una Arquitectura", en Oíza se deriva a la técnica. Más allá del acuerdo entre materiales, que lo hay, el acto de transcribir ideas al proyecto precisa la sintonía en la multiplicidad de sistemas independientes desarrollados por las técnicas y de estos con los sistemas arquitectónicos profundos.

La intuición, la misma que presenta ideas en el proyecto desbordando los caminos de la lógica, se convierte durante el proyecto y la obra en aliada imprescindible para ensayar un estado de lucidez ante la técnica que haga posible esa sintonía.

El arquitecto se presenta entonces como mediador. No como una figura mágica que interpreta una realidad desconocida y misteriosa según su propio arbitrio, sino como un personaje capaz de dominar lo cotidiano (y muchas veces complicado y extenso) de la técnica a la vez que sintoniza con lo que tiene de profundo (y complejo) la proyección del ser humano en el mundo.

Este papel de mediador coincide con la interpretación que Oíza extrae de la definición de arquitectura de Le Corbusier. Una definición en dos partes de las que tradicionalmente, recuerda, se nos ha hurtado la segunda:

"La arquitectura es el juego sabio, correcto y magnífico de los volúmenes bajo la luz; la proporción es aún y exclusivamente el juego sabio, correcto y magnífico de los volúmenes bajo la luz." (CORBUSIER, 1998 pág. 178)

Si la primera de ellas presupone la arquitectura como la gran estructura, la gran organización, la segunda identifica a la arquitectura con la proporción, con el decoro, con la adecuación de esa estructura general a una condición particular.

Es el arquitecto el encargado de mediar entre lo general y lo concreto con las herramientas de la lógica y la intuición. Su misión es la de ordenar, la de dar un sentido, no sólo la de organizar para obtener una determinada finalidad parcial.

Y es cuando se aborda ese decoro, esa particularización, cuando se toca de raíz el tema de la arquitectura. Por ello, se cuida con mimo el detalle, porque en él reside la virtud de la arquitectura.

Por medio del detalle se desvela la intención. El detalle se convierte en lo más delicado de la arquitectura al transcribir al mundo físico la abstracción de una idea. En gran medida depende de la intuición para tomar forma definitiva.

Esta manera de entender la arquitectura se evidencia con mayor intensidad al tratar un tipo tan cercano como la casa.

El propio Le Corbusier, al hablar de ella, mencionaba lo práctico como la labor propia del ingeniero $\mathrm{y}$, sin embargo, una vez superada esa utilidad, atendía a las relaciones que permitían aflorar la emoción plástica como la verdadera tarea del arquitecto.

"Mis ojos miran cualquier cosa que enuncia un pensamiento".

(CORBUSIER, 1998 pág. 123)

Es sencillo seguir este discurso en dos viviendas tan queridas para él como la vivienda que realizó al borde del lago Leman para sus padres o su propio apartamento en el edificio de Porte Molitor en París.

En el primer caso, la relación con el paisaje, cómo se pauta el acercamiento a él para hacerlo significante, hace de esta pequeña casa una obra maestra.

La sucesión de marcos que primero limitan la visión al lago (muro exterior) y después la bloquean completamente (acceso a la vivienda) para, un instante después, de forma desmedida, volver a hacer presente una selección del paisaje a través de una tensa ventana horizontal, se completa con la transición a un exterior delimitado por un muro de mampuesto que recoge el paisaje enmarcado a través de un hueco.

Los dos pasos siguientes, opuestos, completan el estado de conciencia del entorno que ofrece Le Corbusier en esta casa: la cercanía máxima al lago -una vez se elimina cualquier tipo de límite y sólo actúa como tal el borde de la plataforma de asentamiento de la casa- y la máxima distancia -al subir por el exterior a la cubierta ajardinada de la vivienda, que permite medir la casa y al propio habitante con el paisaje lejano-

La transcripción de estos marcos como una sucesión de muros paralelos al borde del lago, provoca la dilatación temporal necesaria para comprender la secuencia en un recorrido zigzagueante que manipula tanto la visión del entorno, como el sonido -dando la espalda a la ruidosa carretera- o la luz -por secuencias de luz y sombra-.

a intuición de este acercamiento al paisaje para crear un lugar, guía la lógica del recorrido que lo transcribe como espacios entre muros con vistas seleccionadas.

En su apartamento de Porte Molitor, lo doméstico cobra mayor importancia y el paisaje se interioriza. Por un lado es el lugar para la invención, para la excepción de la cama elevada hasta la cota justa que permite entrar en relación con el paisaje lejano de cubiertas de París; de la puerta profunda, que se muestra ambigua en su doble condición de puerta y almacenaje; de la pequeña cocina diferenciada en zonas de trabajo por sus muebles y acceso independiente. Pero también es el lugar del paisaje y del recorrido que permite espaciar la zona destinada a estudio de pintura de la dedicada a vivienda; de hacer presente en ese lugar, a través de la subida a la cubierta, la naturaleza exterior del cielo y la luz cenital; en definitiva, de introducir el paso del tiempo y la experiencia de ese tiempo en la transición entre una zona de trabajo y una zona vividera, entre el hombre plástico y el hombre que necesita un descanso.

En este caso, la transcripción de esta intuición desafía a la lógica eficiente del máximo aprovechamiento.

La revolución que estas viviendas supusieron hace casi cien años, hoy día puede considerarse una lección asimilada para la arquitectura.

Sin embargo, el mestizaje que el propio Le Corbusier planteaba entre la lógica y la intuición sigue siendo tan actual como entonces.

En este sentido es en el que se propone concluir esta reflexión con el análisis de otra casa, esta vez contemporánea, para descubrir la manera en que se acercan a ella el arquitecto y el ingeniero.

La vivienda es la Maison à Bordeaux, el arquitecto es Rem Koolhaas y el ingeniero, Cecil Balmond.

La documentación y las publicaciones sobre esta obra son extensas², por lo que se analizará exclusivamente cómo se acercan ambos a la casa como objeto estructural y la interpretación que de ello se puede establecer en relación al encargo del cliente ${ }^{3}$.

La estructura, en su doble cometido de ordenar el edificio y de soportarlo sobre el terreno, es el primer elemento significativo de la vivienda. Además es el que vincula de forma especial a ingeniero y arquitecto.

La renuncia a una estructura convencional (o "eficaz" sólo desde la economía) es el primer paso para lograrlo. La descripción de la estructura permite, además, conocer la vivienda como objeto.

La Villa Lemoine en Floirac, se muestra aparentemente contradictoria desde el principio. 
La casa se conforma por la superposición de espacios en tres niveles paralelos y diferentes: nivel de cielo, nivel de tierra y nivel de cueva. Sin embargo, en la imagen que representa el concepto estructural de la vivienda, ninguno de los elementos que aparecen es un plano horizontal y tampoco se explicitan los niveles (FIG. 1).

Realmente se representa sólo la suspensión de la planta superior, que se muestra como una caja de planos

verticales rígidos con múltiples perforaciones circulares, y el conocido desplazamiento de los puntos de apoyo, que confiere asimetría a la estructura. No es necesario más para resolverlo desde el concepto.

La nota manuscrita, sin embargo, parece acusar el exceso de realidad de representar el bloque que actúa de contrapeso.

Este es un punto de desencuentro con el ingeniero Cecil Balmond, que explica el anclaje de la siguiente manera:

"Conceptually we wanted a very visible reading of "anchor", a rock hanging freely from a cable. Through design development and concerns for safety the rock had turned into a concrete dead weight, located in a pit and held down further by a buried foundation. This was now too expensive. It was cheaper to bury the anchor they said, and have the cable tie disappear into the ground, fixed onto a lump of dead concrete.

This was a pity. The visible counterbalance underlined the drama of tipping over. But savings had to be made, and we reluctantly agreed to the change. Only a plate on the surface would denote passage through to the subterranean holding. It was expedient but not poetic not our daredevil vision of a hung weight in space denoting a danger moment."4

(Balmond, 2002 pág. 43 )

En un croquis de obra (29/09/1996) $)^{5}$, el equipo informa del cambio en la solución inicial de ARUP. Esta consistía en un contrapeso doble, la parte anclada al suelo como receptáculo de $60 \mathrm{~T}$, y una parte de carga muerta colgando del voladizo de 40T. Ambas estarían unidas por lo que parece un nuevo tensor, lo que permitiría hacer visible un mínimo movimiento de esta última (FIG. 2).

Esta elaboración tan complicada parece, por tanto, que fue únicamente opción de la ingeniería. La maqueta de la casa enviada al MoMA ese mismo año 1996 muestra la representación ideal de la roca como un anclaje profundo, no como algo visible para el visitante ${ }^{6}$.

El ingeniero trata el detalle poniendo el énfasis en el objeto particular de la estructura. El detalle (o no detalle) expresado por el arquitecto, intuitivamente vincula la estructura con el habitante de la casa.

Lo que conceptualmente parece indicar Koolhaas es que fuera la Tierra, la gran roca, la que mantuviera el equilibrio de la casa y, con ella, el del señor Lemoine.

A la vista de estos croquis, es fácil tener la impresión de estar ante un ready-made al modo de Duchamp. La utilización de elementos habituales -aquí estructuralespiezas combinadas de forma inédita, provoca la sorpresa ante algo nuevo y en cierto modo misterioso e incomprensible.

Es lo que le ocurre a Guadalupe Acedo, la encargada de la casa que protagoniza el documental "Koolhaas houselife" (Bêka, y otros, 2008): no consigue identificar el modo en el que se sostiene el cuerpo elevado.

No encuentra unos apoyos "tradicionales" y comenta con cierto desasosiego que la casa "está suspendida",
Lo que conceptualmente parece indicar Koolhaas es que fuera

la Tierra, la gran roca, la que

mantuviera el equilibrio de la casa

y, con ella, el del señor Lemoine.

cuestión que le preocupa en los días de viento desde la pieza de dormitorios, porque la casa parece moverse.

A este carácter experimental de la estructura se añade la manipulación aparente de los elementos de apoyo.

Son conocidas las operaciones en relación al cilindro estructural de la escalera de dormitorios - forrado de paneles de acero pulido que reflejan el entorno ajardinado- y la viga asimétrica sobre la que descansa la caja - que se pinta completamente de negro salvo el soporte interior, que es blanco.

Pero la ambigüedad que envuelve la estabilidad de la casa, se completa gracias a otros elementos: la plataforma interior, la puerta inclinada de acero y la carpintería metálica del panel corredero de vidrio. Todos ellos son móviles, y todos, por su dimensión y disposición, son relevantes en la experiencia de la casa.

De la carpintería sólo se perciben los montantes similares en sección a un pilar- al estar embutidos en suelo y techo los travesaños del marco. La puerta inclinada se presenta como un panel en equilibrio inestable, puesto que ambos apoyos también se ocultan.

Al accionarlos se completa el equívoco sobre la forma de soportar el cuerpo elevado. Por un lado, "pilares" que "bailan" en la planta; por otro, planos inclinados en equilibrio imposible para transmitir cargas.

En relación con ellos, la viga sobre el último forjado y el tirante hasta el terreno, se conciben como otros elementos más del mecanismo, cuya misión es activar el espacio del patio.

El conjunto no se cuestiona por la solidez de la caja suspendida. La caja "está" ahí arriba, aunque no se sepa cómo se mantiene.

La plataforma elevadora, con la mesa de despacho, completa la respuesta a la petición del cliente de no crea "una casa simple" sino "una casa compleja, porque será la casa que defina mi mundo"

Con ella se compensa el estado de invalidez en que se encuentra, hasta el punto de que "negar la invalidez" sea el arranque del proyecto.

Aquí será la máquina la que libere al hombre de sus limitaciones.

Funcionalmente, le permite salvar las escaleras para desplazarse por la casa. Espacialmente, le libera de la gravedad. Para él, queda suspendida. La acción de su cuerpo, su paso de una planta a la otra, origina una serie de cambios en la configuración de los límites entre casa y plataforma: activa el espacio de la casa, un espacio que termina de conformarse como en una coreografía lentamente, con su llegada o su partida ${ }^{9}$.
Una vez que muere el señor Lemoine el sentido de la vivienda se pierde, la casa se "arruina". Se cataloga como Monumento Histórico en 2002 y se abre al público.

Hoy puede considerarse un híbrido entre museo, vivienda y fetiche, pero si no se repara en las claves que permiten transcribir las intuiciones del proyecto a la obra final, $\mathrm{s}$ uno no se ata a la silla del propietario, no será capaz de dar el salto que esta casa le ofreció al señor Lemoine. 1'Con motivo de la lección inaugural del curso académico 1991-
1992 en la E.T.S.A.M.

${ }^{2}$ Se recomienda especialmente la monografía de Beatrice Lampariello "Villa à Floirac" (Lampariello, 2011) y el libro-película 2008). Para acceder a fuentes originales de documentación de esta vivienda se ha acudido a los fondos depositados en la Bibliothèque Kandinsky, en París, y a visitar la vivienda durante las Journées Européennes du Patrimoine.

${ }^{3}$ Es necesario recordar la situación de minusvalía del propietario de la casa y la petición expresa de crear una casa que reflejara la

4 "Conceptualmente queríamos una lectura muy visual del "anclaje", una roca que cuelga libremente de un cable. Por el proceso de diseño y los condicionantes de seguridad, la roca se un foso y además retenido por una cimentación oculta. Esto era ya demasiado caro. Dijeron que era más barato enterrar el anclaje y que el atado del cable desapareciera en el suelo, fijado a una masa de hormigón

Fue una pena. La visibilidad del contrapeso subrayaba el drama del vuelco. Sin embargo, había que ahorrar y aceptamos el

cambio a nuestro pesar. Sólo una placa en la superficie remarcaría el paso del cable hacia el soporte subterraneoo. Era eficaz, pero no el espacio señalando un instante de riesgo." Traducción propia del texto original. En lavist.

se constata que el cable penetra en la tierra, a como vivienda el arquitecto. La placa propuesta por el ingeniero ha quedado sepultada.

${ }^{5}$ Bibliothèque Kandinsky, caja KOOL 6, Fax de obra 29/09/1996, 5 páginas.

${ }^{6}$ La maqueta está catalogada como object number 101.1996 en la web del MoMA. Se puede consultar junto con las

inark/97 de la maqueta en hittp://www.moma.org/collection/ de ser en torno a finales de 1994. En un fax de obra fechado deb 17-10-1994 (caja KOOL 4) Jeanne Gang envía la siguiente el Koolhaas: "II. MOMA MODEL: Maison à Floirac. It's urgent to know the results of your conversation with Riley so taht Frank Parthesius can begin with model."

${ }^{7}$ La traducción es del autor. Original en LAMPARIELLO, Beatrice. Villa a Floirac. Rem Koolhaas/OMA 1994-1998, Ed. ARACNE

8 Ídem.

9 El paso de la plataforma requiere de una adaptación de sus límites, tanto por seguridad de utilización de la vivienda como para adaptar los acabados en las zonas de transición.

BIBLIOGRAFÍA

BALMOND, Cecil. 2002. Informal. Munich : Prestel, 2002. 3-79132400-4

BÊKA, lla y LEMOÎNE, Louise. 2008. Koolhaas Houselife. s.l. 978-88-903602-0-6.

CORBUSIER, Le. 1998. Hacia una arquitectura. Barcelona : Apóstrofe, 1998. Vers une architecture. Paris : Crès, 1923. - 1 edición-. ISBN 978-84-455-0277-8.

LAMPARIELLO, Beatrice. 2011. Villa a Floirac. Rem Koolhaas/ OMA

SÁENZ DE OÍZA, Francisco Javier. 1991. Presentación del curso académico 91-92. Nídeo (DVD)] Madrid : Escuela Técnica Superior de Arquitectura, 1991. Lección de Apertura de Curso Académico en la E.T.S.A.M.

\section{ABSTRACT}

El articulo indaga en las relaciones entre transcripción e intuición en el trabajo del arquitecto. La interpretación de Oiza sobre la definicion de arquitectura dada por Le Corbusier muestra la mediación como forma evidente de relación entre los términos propuestos. Este mediar se convierte así en la misión principal del arquitecto. La consideración de dos ejemplos de viviendas desarrolladas tempranamente por Le Corbusier muestra esta aproximación como un alegato en defensa del papel del arquitecto. La actualidad de este acercamiento permite transponerlo al análisis de obras contemporáneas, como la Maison à Bordeaux de Rem Koolhaas. La diferente consideración por parte de arquitecto e ingeniero que se muestra en faxes de obra respecto a un detalle de la vivienda permite reflexionar en este sentido.

PALABRAS CLAVE: Mediación, Oíza, Le Corbusier, Koolhaas, Balmond, Floirac.

ÁLVARO MORENO HERNÁNDEZ es Doctor Arquitecto y Profesor ayudante del Departamento de Proyectos Arquitectónicos de la ETSAM Universidad Politécnica de Madrid (UPM). 